\title{
Une céramique des phénomènes
}

\section{Ceramic of phenomenons}

\author{
Jean Girel ${ }^{1}$ \\ ${ }^{1}$ Artiste, gireljean@orange.fr
}

RÉSUMÉ. Ce que j'appelle mon bestiaire est loin d'une céramique animalière, et encore plus loin d'une céramique décorative à motifs animaliers. II s'agit plutôt d'une céramique des phénomènes, où le sujet animalier est prétexte à la recherche d'équivalents plastiques de nature exclusivement céramique, le sujet étant clairement défini afin qu'il n'y ait pas d'équivoque sur la matière obtenue. C'est donc le contraire d'une céramique du hasard. Le caractère usuel de ces pièces (boîtes, coupes, vases...) est toujours présent, au moins dans le souvenir d'une fonction : préserver, contenir, verser. Ce lien avec un usage éventuel est pour moi presque un problème d'éthique : l'autre fonction que j'en attends est de réveiller la mémoire d'une céramique rituelle, dont le sens s'est perdu avec l'abandon des rites et des mythes, mais dont on peut percevoir parfois un lointain écho. Le bestiaire est la voie que j'ai choisie pour renouer avec le sens primordial de la céramique.

ABSTRACT. What I call my bestiary is neither an animal ceramic nor a ceramic decorated with animal patterns. I would rather call it a ceramic of phenomenons, in which animal is a pretext to find artistic solutions only by means of ceramic. Subject (animal type for instance) is clearly exposed in order to show what is expected. It is therefore the opposite of a ceramic made by chance. The useful aspect of my works (boxes, cups, vases...) is here every time, at least as reminder of a function : to preserve, to contain, to pour. The link with a potential use of a ceramic is a matter of ethic : I also expect it to be able to revive the memory of a ritual ceramic, of which meaning disappeared as myths were abandoned but could still be found as an echo. Bestiary is the way I chose to go back to the primordial sense of ceramic.

MOTS-CLÉS. usage, rituel, bestiaire, épiderme, carapace, phénomènes, fusion, ébullition, viscosité, tension superficielle.

KEYWORDS. use, ritual, bestiary, epidermis, shell, penomenons, molten, boiling, viscosity, superficial tension.

En guise d'introduction, Je souhaiterais distinguer les deux notions portées par le titre de l'exposition Formes vivantes et dire en quoi mon travail est lié à chacune d'entre elle.

Le vivant tout d'abord, peut être schématiquement associé à tout ce qui se développe dans les températures de l'eau, entre sa congélation et son ébullition, avec l'aide du carbone. Faut-il s'arrêter d'emblée à l'idée qu'un créateur de pièces passées dans la fournaise, à des températures censées détruire toute forme de vie n'a rien à voir avec cette notion ? Ou bien plutôt considérer que le travail du céramiste, qui se situe dans les températures de fusion des silicates, entre état solide et phase vapeur, dans une échelle géologique, relève d'une autre forme du vivant?

Vient ensuite la question de la forme : j'ai décidé en 1975, après avoir été fasciné par deux céramiques chinoises de la dynastie Song, un bol fourrure de lièvre et une urne funéraire en céladon, de quitter définitivement la peinture, qui fait appel à l'esprit (cosa mentale) au profit de la pratique céramique, qui engage plutôt le corps et les sens aux prises avec la matière.

La céramique est un art d'abord rituel (comme peuvent en témoigner la Vénus de Dolni Vestonice, la poterie japonaise de l'époque Jomon, l'art précolombien ou encore les céramiques chinoises jusqu'à la dynastie Song) avant de se destiner à l'usage. Je revendique dans ma pratique ces deux fonctions :

C'est L'usage qui détermine la forme du pot : contenir, verser, cuire, conserver déterminent la forme le bol, la coupe, le bassin, la boîte et bien d'autres encore se chargent d'une valeur symbolique d'accueil, d'offrande, de préservation. La fonction crée l'organe et la typologie potière 
montre bien le rapport qu'elle entretient avec le vivant, avec le corps : le col, le bec, la lèvre, le goulot, le ventre, le flanc, le pied, le talon, le cul des pièces !

On ne peut pas parler en poterie d'invention de la forme, mais plutôt d'une éclosion, d'une croissance, d'une poussée.

Quand au caractère rituel de ce que j'appelle mon bestiaire, il est assuré par une inflexion de la forme, un ornement discret, un accessoire de préhension qui évoque un animal, une spirale qui évoque un coquillage.

Ce n'est donc pas la forme qui constitue ma préoccupation, même si j'attache le plus grand soin à sa naissance, toujours sur le tour, c'est plutôt ce qui va se passer après, dans le four, c'est-à-dire à la création d'une pièce qui peut évoquer un animal, un insecte, son épiderme ou sa carapace, par des moyens spécifiquement céramiques, en m'interdisant tout usage du dessin ou du pinceau, en demandant à la vie de la matière dans le feu de créer elle-même couleurs, matières et textures aptes à évoquer le sujet.

Quelques photos prises dans mon bestiaire peuvent illustrer ma conception de la forme, et de sa nécessaire discrétion :

L'œuf, contenant du vivant en formation par excellence :

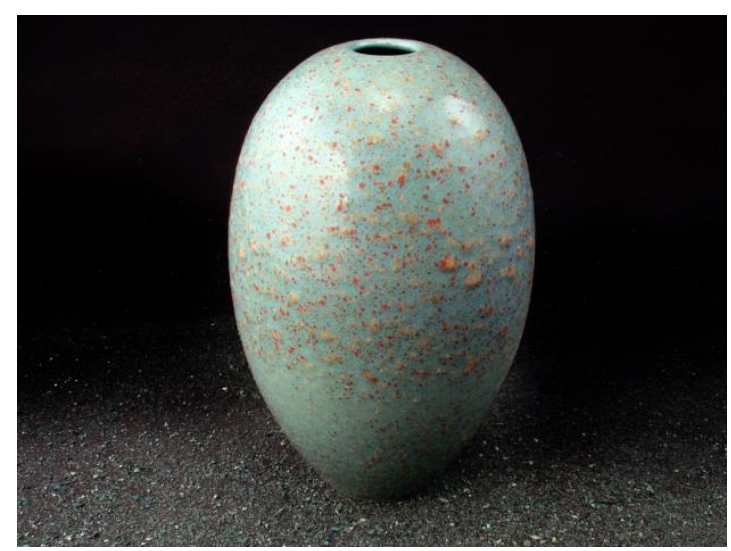

Jean Girel, Oeuf, porcelaine

Le coquillage, qui naît d'une spirale comme l'argile sur le tour en rotation :

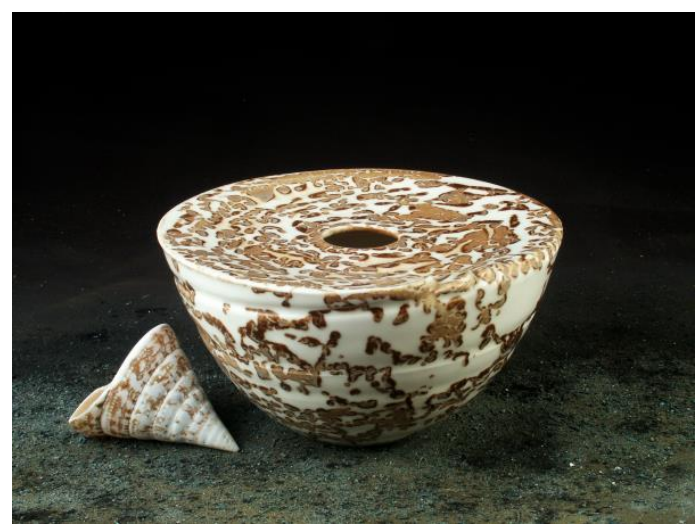

Jean Girel, vase coquillage, porcelaine

Le bassin, le bol, la coupe, la boîte, qui peut devenir urne ou tambour de pluie, qui peut receler une mémoire ou convoquer la pluie : 


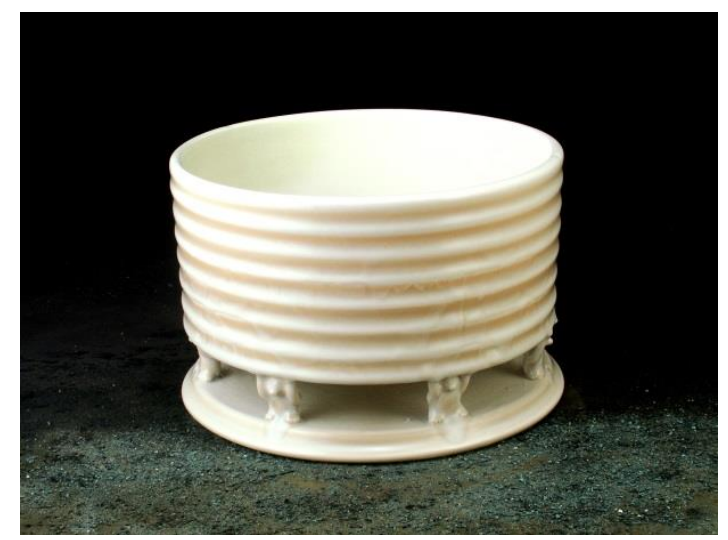

Jean Girel, bassin, porcelaine

Pour tenter de définir la spécificité de ma recherche, j'ai choisi dans mon bestiaire un thème partagé par d'autres céramiques présentes dans l'exposition Formes vivantes, le thème de la grenouille.

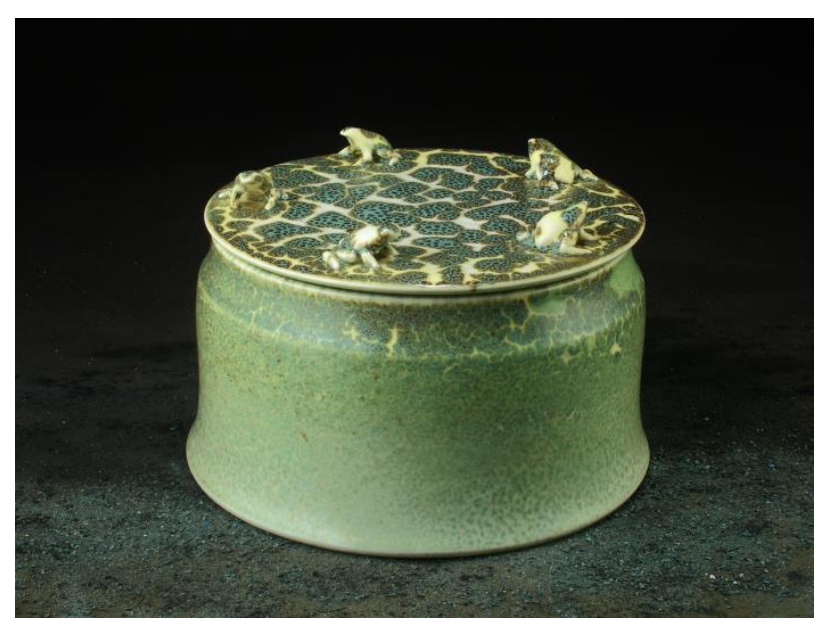

Jean Girel, boîte grenouilles, porcelaine

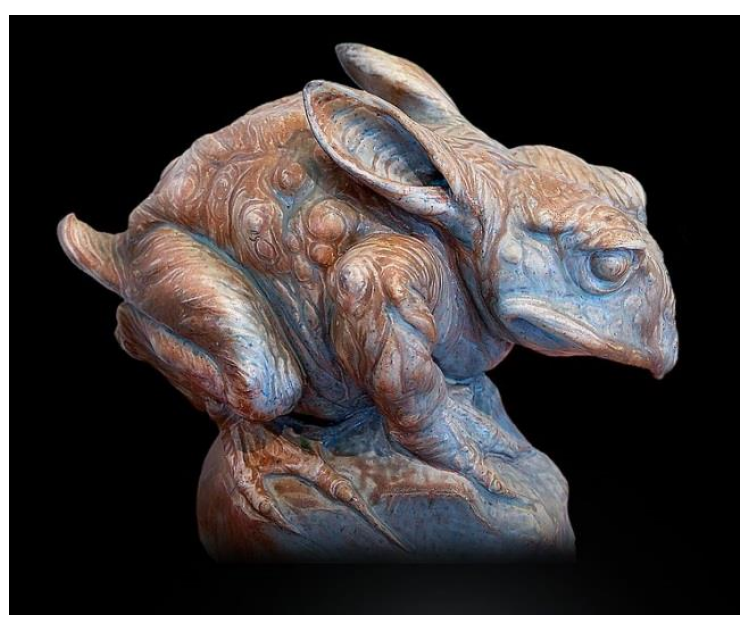

Jean Carriès, Grenouilles à oreilles de lapin, grès émaillé, 1891, Paris Petit Palais, photo : Wikimedia commons

Jean Carriès, ce météore de la céramique, avait une prédilection pour les batraciens et était fasciné par la texture de leur épiderme. Les couvertes qu'il a créées pour les imiter montrent également sa fascination pour la transmutation des matières par le feu. C'est ce double intérêt qui l'a engagé, en tant que sculpteur, à une transmutation de la forme même du batracien, qui devient grenouille à oreilles de lapin, ou « grenouillard», être hybride mi-homme, mi-animal. Créateur de matières, il agit aussi en sculpteur créateur de formes.

Bernard Palissy aussi est fasciné par le monde des batraciens, omniprésents dans son œuvre aux côtés des reptiles. Pour les représenter, il utilise le moulage, le modelage, la peinture, écaille après écaille, tache après tache, à l'aide de pigments broyés à froid. Il agit en peintre, en sculpteur, en graveur, le feu étant simplement chargé de fixer ce qui a été préparé à froid, à l'état plastique, à l'état humide. Huguenot intransigeant, pourfendeur des théories alchimistes, « qui cherchent la génération des métaux par le feu », donc à édifier par le destructeur, pour lui « le commencement et origine de toute chose naturelle est eau. » Palissy se méfie du feu qu'il considère comme diabolique. 

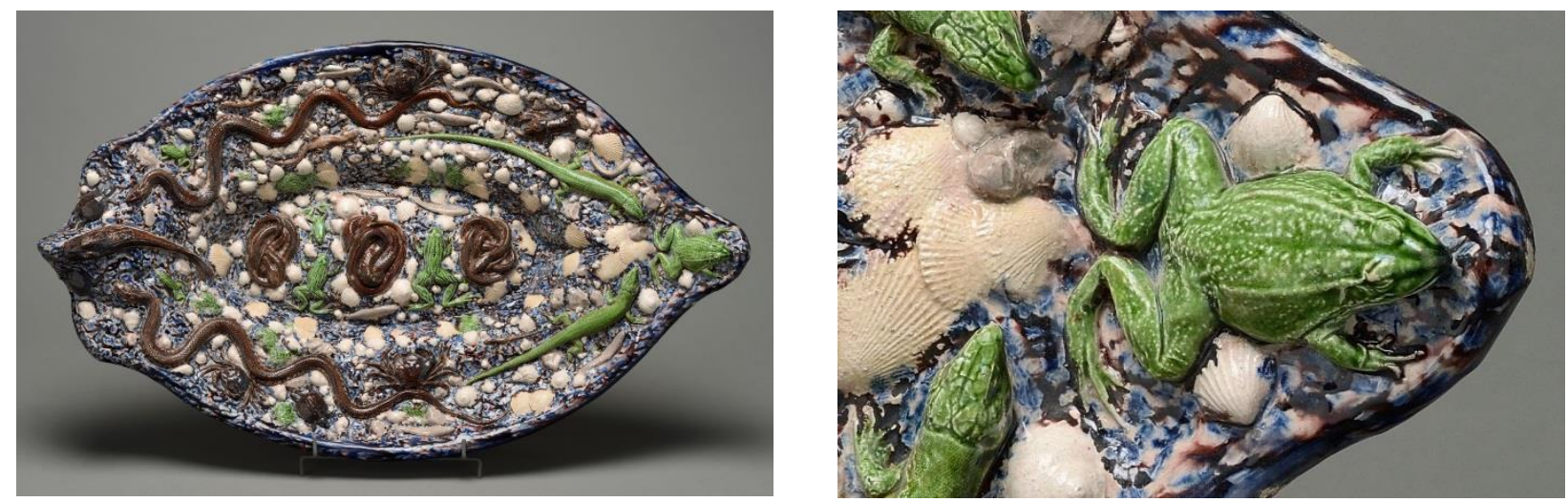

Plat, Atelier de Bernard Palissy (Attribué), seconde moitié du XVle siècle, Terre argileuse, galcçure plombifère, Lyon Musée des Beaux-Arts, Photo : Alain Basset.

Frère Daniel de Montmollin, qui est cité dans le catalogue de l'exposition, et qui a fait un travail fondamental sur l'usage des cendres végétales comme constituant principaux des couvertes de haute température, parle justement à leur sujet d'une nouvelle biologie liée à l'incandescence. Il reste néanmoins très prudent aussi vis-à-vis du feu, écrivant que lorsque le potier emmure ses céramiques dans son four, il « les livre à l'arcane d'un autre opérateur ».
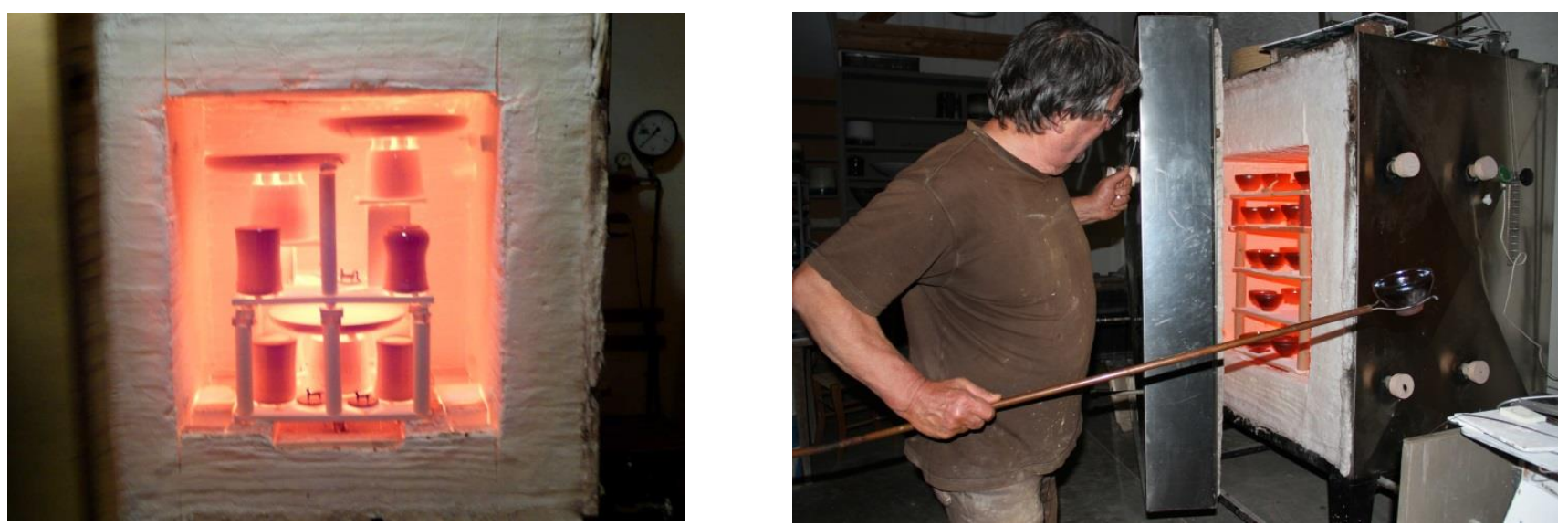

Jean Girel dans son atelier

Personnellement, dès mes débuts j'ai déjà tenté d'inverser cette soumission au feu, destructeur selon les uns, pythie selon les autres, cette tyrannie du four que l'on a pas le droit de déranger. J'ai construit dix-huit fours pour mon usage personnel et tous comportaient une porte permettant, en l'ouvrant en cours de cuisson, d'observer pour mieux les comprendre et les maîtriser tous les phénomènes qu'accompagnent la montée et la descente en température.

Comme j'ai pu l'expliquer en introduction, je m' 'interdis tout travail de dessin ou de peinture sur mes pièces. Je suis donc allé à la recherche de solutions capables de créer d'elles-mêmes des motifs, des textures, des détails, pendant la cuisson.

Ce n'est pas dans les livres de céramique que j'ai pu les découvrir, mais plutôt partout où la curiosité me pousse : dans la nature, dans les livres de cuisine (les états du sucre, bien décrits, sont une source inépuisable d'idées pour le céramiste). En réalité, les phénomènes de la nature sont toujours les mêmes : entre le caramel et une couverte en phase de refroidissement, il y a simplement un changement d'échelle.

Alors, comment vais-je faire pour donner un équivalent plastique et pictural de lépiderme de la grenouille, sans dessin, sans pinceau, en posant simplement des couvertes les unes sur les autres sur la pièce ? 
Il y a des plages de couleur verte sur un fond plus clair, des ocelles à l'intérieur de ces plages. Sous le ventre des batraciens, on observe souvent un réseau petits globules arrondis : comment restituer toute cette complexité en l'espace d'une cuisson.

\section{Les retirements}
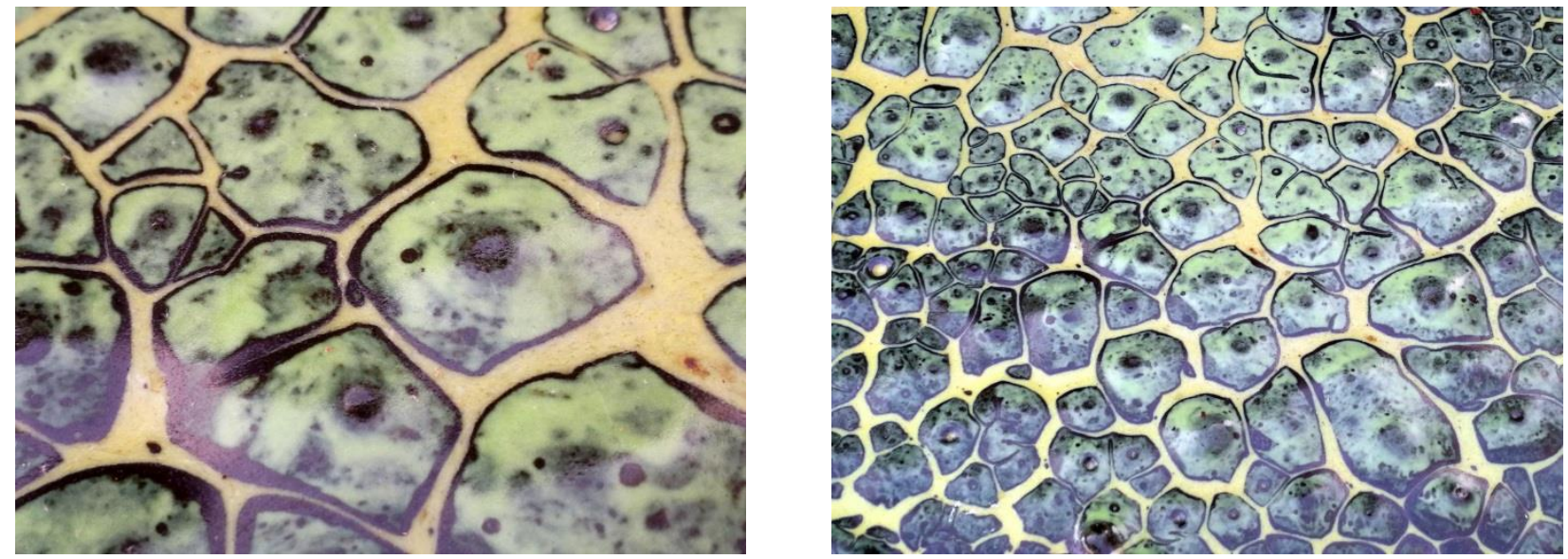

Jean Girel, Boîte grenouilles, porcelaine, (détail)

C'est un phénomène que l'on observe quand une flaque de boue sèche au soleil. Plus l'argile qu'elle contient est colloïdale, donc capable de fixer beaucoup d'eau autour de ses particules, plus le retrait sera important. En superposant une couverte surbroyée, donc très colloidale sur une autre qui ne l'est pas, le phénomène de retrait se déclenche dès le séchage puis en début de cuisson. On peut même superposer le phénomène pour obtenir un réseau fin sur des plages plus étendues.Le dessin de contour des plages peut aussi être contrôlé, pour obtenir des plages hexagonales, arrondies, carrées ou rondes.

\section{Les ocelles}

À l'intérieur de ces plages on peut faire émerger des taches de couleur différentes, des ocelles, voire des pustules. L'effet est ici produit par un phénomène d'ébullition d'une couche sous-jacente. J'ai beaucoup travaillé sur ce phénomène en étudiant le volcanisme à l'île de la Réunion et en recherchant les couvertes à gouttes d'huile des potiers Song.

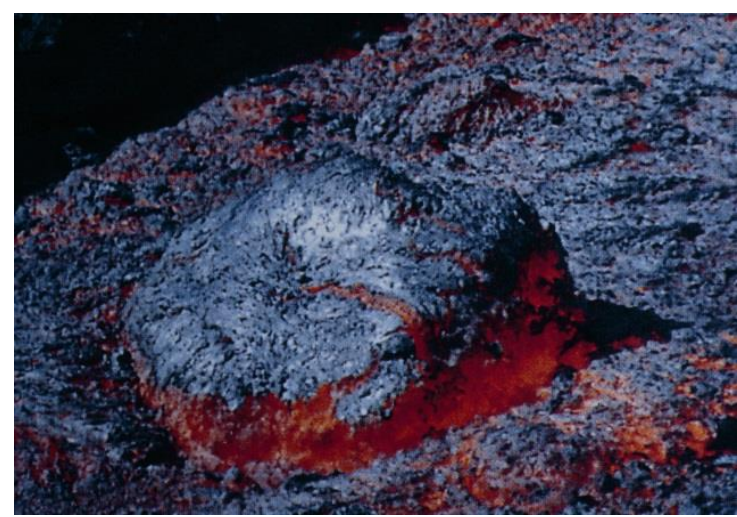

Détail d'une éruption volcanique, île de la Réunion

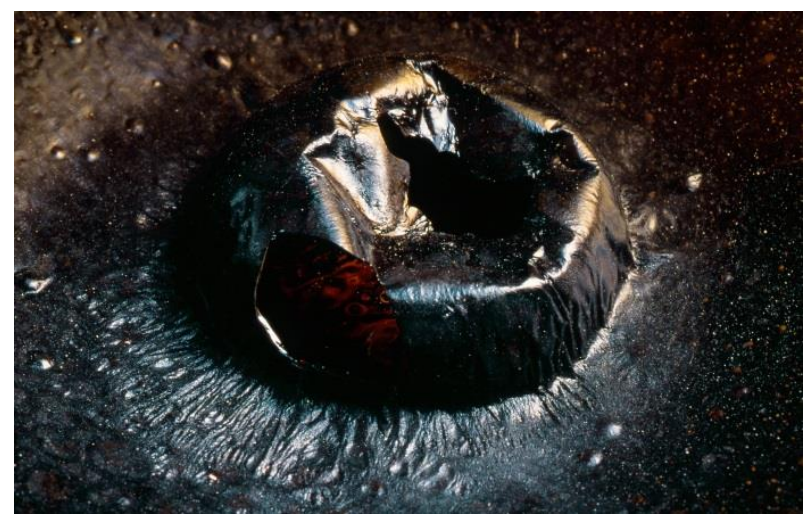

Détail d'ébullition d'une pièce " gouttes d'huile » figée par sa sortie du four à $1320^{\circ} \mathrm{C}$ 


\section{Décollement et viscosité}

Si la couche superficielle a peu d'adhérence à sec, mais une forte viscosité à haute température, des fragments de couverte peuvent partiellement se détacher et s'agglomérer autour d'un point fixe, créant des plages aléatoires.
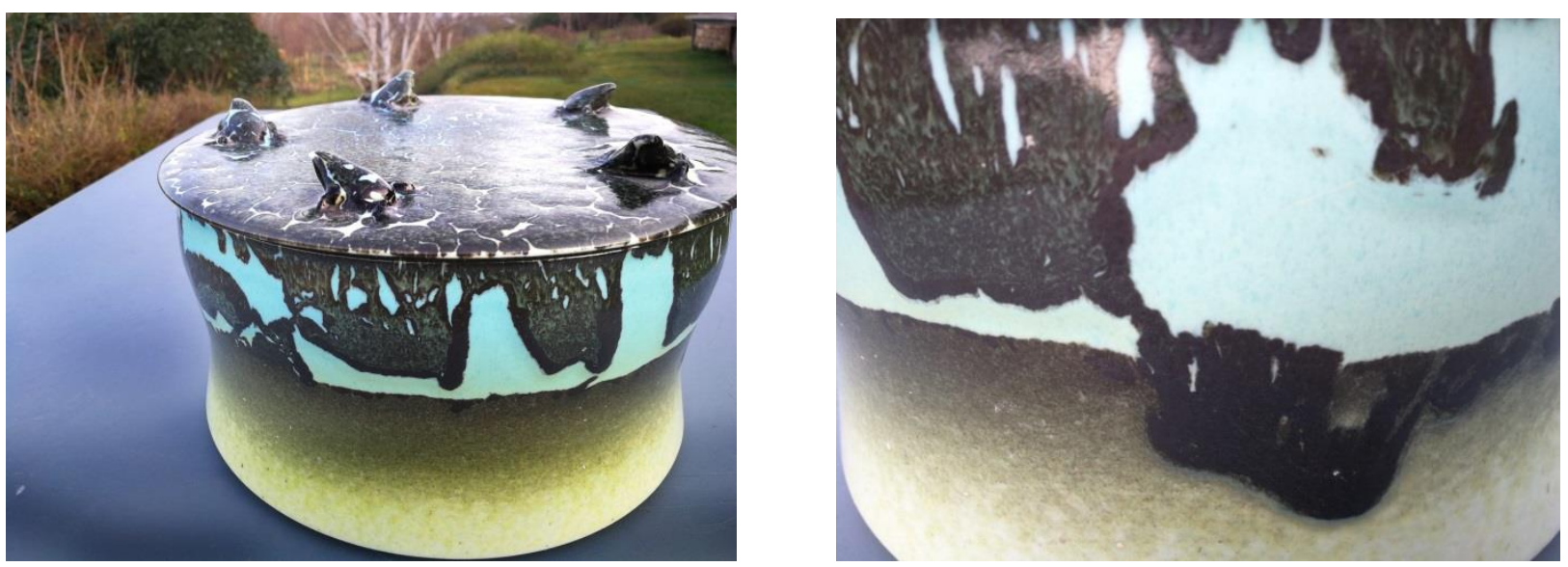

Jean Girel, Boîte grenouilles, porcelaine, (détail)

\section{Texture de galuchat du ventre des grenouilles}

Pour obtenir cet effet, j'ai fait appel à un phénomène mis en œuvre lorsque je cherchais à imiter la matière de certains champignons de la famille des lycoperdons. La piste m'avait été donnée lors d'une rêverie le long de ma mare précisément un jour d'observation de grenouilles. Des gouttes d'eau dansaient sur les feuilles de lotus. Pourquoi l'eau se comportait-elle comme du mercure à la surface des ces feuilles ? C'est en lisant Gilles de Gennes que je trouvais la clé de l'énigme : la feuille était hydrophobe (elle refusait de se laisser mouiller), et la tension superficielle de l'eau prenait alors le dessus sur le nappage. J'imaginais alors une couverte hydrophobe, donc d'une texture particulière, et une autre, à forte tension superficielle, et le tour était joué.

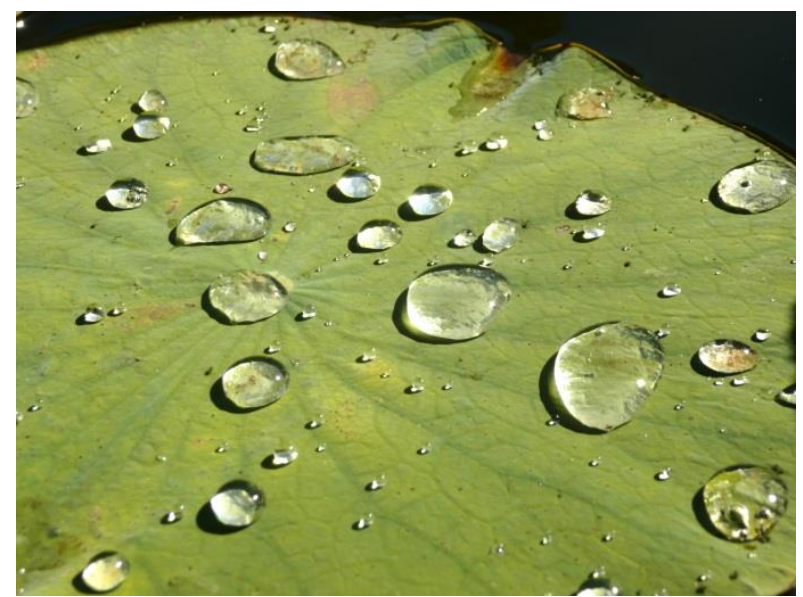

Gouttes d'eau sur une feuille de nénuphar

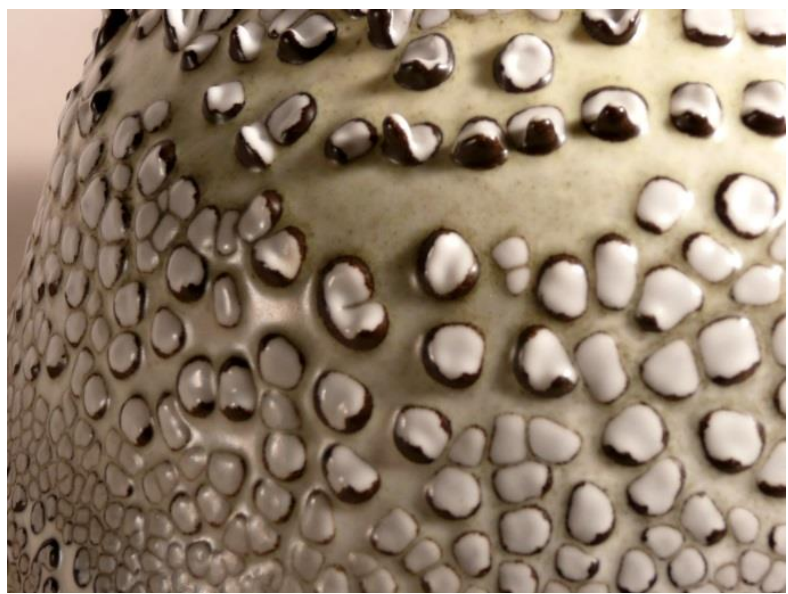

Jean Girel, Boîte grenouilles, porcelaine

\section{Conclusion}

En travaillant de cette façon, la céramique apparaît comme un jeu, un jeu complexe où l'on prémédite une sorte de programmation : chaque phénomène doit nécessairement se produire ; leur conjonction doit assurer l'unité du sujet abordé et en même temps provoquer un espace de liberté, que l'on pourrait appeler le hasard. L'inspiration (si l'on peut employer ce terme) est alors partout, 
dans la mare, dans le volcan, au moment où l'on fait la cuisine, ou quand on regarde passer les nuages.

L'idée que l'homme est ajouté à la nature (Ars homo additus naturae) pour mieux la dominer, idée qui va de la Bible à Marx en passant par Descartes qui pense l'homme comme maitre et possesseur de la nature est en train de produire les effets que l'on sait. Le sentiment de communion avec les éléments, la découverte ou redécouverte émerveillée de la nature me paraît plus féconde et nécessaire pour affronter l'avenir.

C'est pourquoi j'ai écrit en exergue de mon dernier cahier d'atelier cette phrase du grand géographe Elysée Reclus, poète et philosophe libertaire, écologiste prophétique avant la lettre : «l'homme est la nature prenant conscience d'elle-même». 